PROCEEDINGS OF THE

AMERICAN MATHEMATICAL SOCIETY

Volume 126, Number 2, February 1998, Pages 375-382

S $0002-9939(98) 04428-1$

\title{
A POINTWISE SPECTRUM AND REPRESENTATION OF OPERATORS
}

\author{
N. BERTOGLIO, SERVET MARTÍNEZ, AND JAIME SAN MARTÍN \\ (Communicated by Palle E. T. Jorgensen)
}

\begin{abstract}
For a self-adjoint operator $A: H \rightarrow H$ commuting with an increasing family of projections $\mathcal{P}=\left(P_{t}\right)$ we study the multifunction $t \rightarrow \Gamma^{\mathcal{T}}(t)=$ $\bigcap\left\{\sigma_{I}: I\right.$ an open set of the topology $\mathcal{T}$ containing $\left.t\right\}$, where $\sigma_{I}$ is the spectrum of $A$ on $P_{I} H$. Let $m_{\mathcal{P}}$ be the measure of maximal spectral type. We study the condition that $\Gamma^{\mathcal{T}}$ is essentially a singleton, $m_{\mathcal{P}}\left\{t: \Gamma^{\mathcal{T}}(t)\right.$ is not a singleton $\}=0$. We show that if $\mathcal{T}$ is the density topology and if $m_{\mathcal{P}}$ satisfies the density theorem, in particular if it is absolutely continuous with respect to the Lebesgue measure, then this condition is equivalent to the fact that $A$ is a Borel function of $\mathcal{P}$. If $\mathcal{T}$ is the usual topology then the condition is equivalent to the fact that $A$ is approched in norm by step functions $\sum_{n \in \mathbb{N}} \Gamma^{\mathcal{T}}\left(\alpha_{n}\right)\left\langle P_{I_{n}} f, f\right\rangle$, where the set of intervals $\left\{I_{n}: n \in \mathbb{N}\right\}$ covers the set where $\Gamma^{\mathcal{T}}$ is a singleton.
\end{abstract}

\section{INTRODUCTION}

Let $H$ be a real separable Hilbert space and $\mathcal{P}=\left(P_{t}: t \in \mathbb{R}\right)$ be an increasing right continuous family of projections, with $P_{-\infty}=0, P_{\infty}=I$ the identity. If $B$ is a Borel real set we denote by $P_{B}$ the projection $P_{B}=\int_{B} d P_{t}$.

For $f \in H$ denote by $m_{f}$ the Borel measure induced by the spectral family $\mathcal{P}$ :

$$
m_{f}(B)=\left\langle P_{B} f, f\right\rangle \text { for any Borel set } B \text {. }
$$

There exists a measure $m_{\mathcal{P}}$, associated to some element of $H$ of maximal spectral type, i.e. $m_{f}<<m_{\mathcal{P}}$ for all $f \in H$. We shall assume that $m_{\mathcal{P}}(\mathbb{R})=1$.

We shall denote by $\mathcal{L}_{\mathcal{P}}$ the completion of the Borel $\sigma$-field with respect to $m_{\mathcal{P}}$. If $G \in \mathcal{L}_{\mathcal{P}}$ there is a Borel set $B$ such that $m_{\mathcal{P}}(G \Delta B)=0$, and we define

$$
P_{G}:=P_{B}
$$

This definition is consistent because if $B_{1}=B_{2} m_{\mathcal{P}}$-a.e., then $P_{B_{1}}=P_{B_{2}}$; in fact

$$
\left\|\left(P_{B_{1}}-P_{B_{2}}\right) f\right\|^{2}=\int_{B_{1} \Delta B_{2}} d\left\langle P_{t} f, f\right\rangle=\int_{B_{1} \Delta B_{2}} \frac{d\left\langle P_{t} f, f\right\rangle}{d m_{\mathcal{P}}(t)} d m_{\mathcal{P}}(t)=0 .
$$

We shall denote by $F_{\mathcal{P}}$ the support of $m_{\mathcal{P}}$; that is, $F_{\mathcal{P}}$ is the smallest closed set of full measure.

Received by the editors July 20, 1995 and, in revised form, April 30, 1996.

1991 Mathematics Subject Classification. Primary 47A11, 47D15.

(C)1998 American Mathematical Society 
Let $A$ be a self-adjoint operator commuting with $\mathcal{P}$. Then $A$ acts on the space $P_{\Lambda} H$ for any $\Lambda \in \mathcal{L}_{\mathcal{P}}$, i.e. $A P_{\Lambda} H \subset P_{\Lambda} H$. Denote

$$
\sigma_{\Lambda}=\text { spectrum of } A P_{\Lambda} \text { on } P_{\Lambda} H
$$

Then $\sigma_{\Lambda}$ is a compact set included in $[-\|A\|,\|A\|]$. If $\Lambda_{1} \supseteq \Lambda_{2}$ and $m_{\mathcal{P}}\left(\Lambda_{2}\right)>0$, then $\sigma_{\Lambda_{1}} \supseteq \sigma_{\Lambda_{2}}$. In fact, if $\lambda \in \sigma_{\Lambda_{2}}$, then there is a sequence $\left(f_{n}\right) \subseteq P_{\Lambda_{2}}(H)$ with $\left\|f_{n}\right\|=1$ such that $\left\langle A P_{\Lambda_{2}} f_{n}, f_{n}\right\rangle \rightarrow \lambda$, from which the property follows.

Our work is concerned with integral representation of a self-adjoint operator $A$ commuting with $\mathcal{P}$. In this context a set of necessary and sufficient conditions is given in [1] and [5].

First let us reduce the problem to the case of continuous $\mathcal{P}$. If $A$ commutes with $\mathcal{P}$ then it commutes with any $\Delta P_{t}=P_{t}-P_{t-} \neq 0$. A necessary condition in order that the operator $A$ can be written in the form $A=g\left(\int s d P_{s}\right)$ is that $A \Delta P_{t}=\lambda_{t} \Delta P_{t}$ for some real $\lambda_{t}$. Denote by $L$ the Hilbert space generated by $\left\{\Delta P_{t} H: t \in \mathbb{R}\right\}$, and let $H^{\prime}=H \Theta L$. The projections $P_{t}$ and $P_{H^{\prime}}$ commute, the family of projections $\mathcal{P}^{\prime}=\left(P_{t}^{\prime}=P_{t} P_{H^{\prime}}: t \in \mathbb{R}\right)$ is continuous, and the restriction $A^{\prime}=A P_{H^{\prime}}$ commutes with $\mathcal{P}^{\prime}$. We have that $A=g\left(\int s d P_{s}\right)$ iff $A \Delta P_{t}=\lambda_{t} \Delta P_{t}$ for all $t$, and $A^{\prime}=g^{\prime}\left(\int s d P_{s}^{\prime}\right)$. Then we can assume $\mathcal{P}=\left(P_{t}: t \in \mathbb{R}\right)$ is continuous.

Since $\mathcal{P}$ is continuous, the measure of maximal spectral type $m_{\mathcal{P}}$ is non-atomic.

Following [6], a sequence $\left\{K_{n}\right\}$ of $\mathcal{L}_{\mathcal{P}}$ sets is said to converge to $t \in \mathbb{R}$ if: $t \in$ $\bigcap K_{n}, m_{\mathcal{P}}\left(K_{n}\right)>0$ and $m_{\mathcal{P}}\left(K_{n}\right) \underset{n}{\longrightarrow} 0$. Let $\mathcal{K}$ be a collection of sequences in $\mathcal{L}_{\mathcal{P}}$.

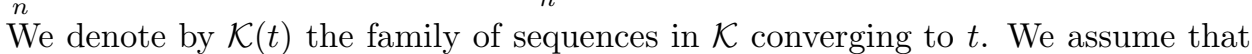
$\mathcal{K}(t) \neq \phi$ for every $t \in \mathbb{R}$.

The upper outer density of $E \subseteq \mathbb{R}$ at the point $t$ is defined by

$$
\bar{D}^{*}(E, t)=\sup \left\{\varlimsup_{n} \frac{m_{\mathcal{P}}^{*}\left(E \cap K_{n}\right)}{m_{\mathcal{P}}\left(K_{n}\right)} /\left\{K_{n}\right\} \in \mathcal{K}(t)\right\} .
$$

The lower outer density $\underline{D}^{*}(I, t)$ is defined as

$$
\underline{D}^{*}(E, t)=\inf \left\{\underline{\underline{\lim }} \frac{m_{\mathcal{P}}^{*}\left(E \cap K_{n}\right)}{m_{\mathcal{P}}\left(K_{n}\right)} /\left\{K_{n}\right\} \in \mathcal{K}(t)\right\} .
$$

If $E$ is $\mathcal{L}_{\mathcal{P}}$-measurable and $\underline{D}^{*}(E, t)=\bar{D}^{*}(E, t)=1$, then we say that $t$ is an (outer) density point for $E$. The density topology $\mathcal{T}_{D}$ is the set of all $I \subseteq \mathbb{R}$ such that $\forall t \in I \quad \bar{D}^{*}\left(I^{c}, t\right)=0$. In this context also see [3], [7] and [8].

We shall assume that $m_{\mathcal{P}}$ satisfies a density theorem; this means that there exists a collection $\mathcal{K}$ such that, for every set $A \subseteq \mathbb{R}$, almost every point with respect to $m_{\mathcal{P}}$ of $A$ is an outer density point for $A$. Under this condition every open set $I \in \mathcal{T}_{D}$ is $\mathcal{L}_{\mathcal{P}}$-measurable, and every point $t \in I$ is a density point of $I$. Moreover a function $\varphi \in \mathcal{L}_{\mathcal{P}}$ is $\mathcal{T}_{D} / \mathcal{T}_{0}$-continuous $m_{\mathcal{P}}$-a.e., where $\mathcal{T}_{0}$ in the usual topology on $\mathbb{R}$. Therefore, if $\varphi$ is a bounded $\mathcal{L}_{\mathcal{P}}$-measurable function, then for $m_{\mathcal{P}}$-a.a. $t$, if $\left\{\mathcal{K}_{n}\right\} \in \mathcal{K}(t)$

$$
\lim _{n \rightarrow \infty} \frac{\int_{K_{n}} \varphi d m_{\mathcal{P}}}{m_{\mathcal{P}}\left(K_{n}\right)}=\varphi(t) .
$$

If $m_{\mathcal{P}}$ is absolutely continuous with respect to the Lebesgue measure, then $m_{\mathcal{P}}$ satisfies the density theorem with respect to $\mathcal{K}(t)=$ class of all regular intervals converging to $t$. 
In the next four lemmas we shall consider a topology $\mathcal{T}$ on $F_{\mathcal{P}}$. Mainly we are interested in the traces of $\mathcal{T}_{D}$ and $\mathcal{T}_{0}$ over $F_{\mathcal{P}}$, which we still denote by $\mathcal{T}_{D}$ and $\mathcal{T}_{0}$. We shall assume that $\mathcal{T}$ fulfills the following two conditions:

(i) $\mathcal{T} \subseteq \mathcal{L}_{\mathcal{P}}$

(ii) $\forall \phi \neq I \in \mathcal{T} \quad m_{\mathcal{P}}(I)>0$.

These two conditions are satisfied by $\mathcal{T}_{D}$ and $\mathcal{T}_{0}$. For $\mathcal{T}_{D}$ they follow respectively from Corollary 4.4 and Corollaries 4.12 and 4.13 of [6]. As for $\mathcal{T}_{0}$, (i) is inmediate and (ii) follows from the definition of $F_{\mathcal{P}}$.

We denote by $\mathcal{I}^{\mathcal{T}}(t)=\{I \in \mathcal{T}: t \in I\}$ the set of open neighbourhoods of $t \in F_{\mathcal{P}}$.

Define the pointwise spectrum of $A$ with respect to $\mathcal{P}$ and the topology $\mathcal{T}$ by

$$
\Gamma^{\mathcal{T}}(t)=\bigcap_{I \in \mathcal{I}^{\mathcal{T}}(t)} \sigma_{I}
$$

for $t \in F_{\mathcal{P}}$.

In the proof of the lemmas we abbreviate $\Gamma(t)=\Gamma^{\mathcal{T}}(t)$ and $\mathcal{I}^{\mathcal{T}}(t)=\mathcal{I}(t)$

Lemma 1. $\Gamma^{\mathcal{T}}(t)$ is a non-empty $\mathcal{T}_{0}$-compact set included in $[-\|A\|,\|A\|]$.

Proof. The sets $\sigma_{I}$ are non-empty and compact, so it suffices to show that the family $\left(\sigma_{I}\right)_{I \in \mathcal{I}(t)}$ has the finite intersection property. This holds because this family is a net with respect to the order induced by inclusion; in fact if $I_{1}, \ldots, I_{n}$ belong to $\mathcal{I}(t)$ the open set $\bigcap_{i=1}^{n} I_{i}$ also belongs to $\mathcal{I}(t)$, and $\bigcap_{i=1}^{n} \sigma_{I_{i}}$ contains $\sigma_{\bigcap_{i=1}^{n} I_{i}}$.

The mapping $\Gamma: F_{\mathcal{P}} \rightarrow$ Subsets $\mathbb{R}$ is a multifunction with compact values. We recall that for $X$ and $Y$ topological spaces, a multifunction $G: X \rightarrow \operatorname{Subsets} Y$, is said to be upper semi-continuous if for all $t \in X$ and for all open set $V$ containing $G(t)$, there exists a neighbourhood $U$ of $t$ such that $G(s) \subset V$ for all $s \in U$ (see $[2])$. Now, set

$$
S(G)=\left\{t \in F_{\mathcal{P}}: G(t) \text { is a singleton }\right\}
$$

and assume $S(G) \neq \varnothing$. When $t \in S(G)$ we identify the singleton $G(t)$ with its unique element. If $G$ is an upper semi-continuous multifunction, then $G: S(G) \rightarrow Y$ is a continuous function.

Lemma 2. $\Gamma^{\mathcal{T}}$ is a $\mathcal{T} / \mathcal{T}_{0}$ upper semi-continuous multifunction.

Proof. Let $V \in \mathcal{T}_{0}$ be an open set such that $\Gamma(t) \subset V$. Since the family of $\mathcal{T}_{0^{-}}$ compact sets $\left\{\sigma_{I}\right\}_{I \in \mathcal{I}(t)}$ is a net with the order induced by inclusion, it is easy to prove that there exists $I \in \mathcal{I}(t)$ with $\sigma_{I} \subseteq V$. In particular, for all $s \in I$ we have $\Gamma(s) \subseteq \sigma_{I} \subseteq V$.

This result implies that there exist measurable selections $\gamma \in \Gamma$, i.e. an $\mathcal{L}_{\mathcal{P}^{-}}$ measurable function $\gamma: F_{\mathcal{P}} \rightarrow \mathbb{R}$ such that $\gamma(t) \in \Gamma^{\mathcal{T}}(t)$ for all $t \in F_{\mathcal{P}}$. Moreover, the functions below are two measurable selections:

$$
\psi_{\Gamma^{\mathcal{T}}}(t)=\max \left\{u \in \Gamma^{\mathcal{T}}(t)\right\}, \quad \phi_{\Gamma^{\mathcal{T}}}(t)=\min \left\{u \in \Gamma^{\mathcal{T}}(t)\right\} .
$$

More precisely:

Lemma 3. $\psi_{\Gamma^{\mathcal{T}}}$ is $\mathcal{T} / \mathcal{T}_{0}$ upper semi-continuous and $\phi_{\Gamma^{\mathcal{T}}}$ is $\mathcal{T} / \mathcal{T}_{0}$ lower semicontinuous.

Proof. This follows from Lemma 2. In fact, if $t \in \psi_{\Gamma}^{-1}(-\infty, r)$, then $\Gamma(t) \subset$ $(-\infty, r)=V$ and there exists a neighbourhood $U$ of $t$ such that $\Gamma(s) \subset(-\infty, r)$ 
for all $s \in U$, so $U \subset \psi_{\Gamma}^{-1}(-\infty, r)$. Hence $\psi_{\Gamma}^{-1}(-\infty, r) \in \mathcal{T}$ and $\psi_{\Gamma}$ is upper semi-continuous. The proof is analogous for $\phi_{\Gamma}$.

Lemma 4. $S\left(\Gamma^{\mathcal{T}}\right)=\left\{t: \Gamma^{\mathcal{T}}(t)\right.$ is a singleton $\}$ is $\mathcal{L}_{\mathcal{P}}$-measurable.

Proof. This follows inmediately from the equality $S(\Gamma)=\left\{t: \psi_{\Gamma}(t)=\phi_{\Gamma}(t)\right\}$.

Lemma 5. Assume $m$ is a non-atomic Borel measure and $\mathcal{J}=\{J\}$ is a family of open intervals covering a Borel set $E$. Then there exists a countable class of disjoint open intervals $\mathcal{I}=\{I\}$ subordinated to $\mathcal{J}$ (i.e. for any $I \in \mathcal{I}$ there exists $J \in \mathcal{J}$ such that $I \subset J)$ satisfying $m\left(E \backslash \bigcup_{I \in \mathcal{I}} I\right)=0$.

Proof. By Lindelöf's theorem there exists a countable class $\left\{J_{n}: n \in \mathbb{N}\right\} \subset \mathcal{J}$ such that $E \subset \bigcup_{n \in \mathbb{N}} J_{n}$. Now define $I_{1}^{\prime}=J_{1}, I_{k}^{\prime}=J_{k} \backslash \bigcup_{i<k} J_{i}$ for $k>1$. It is easily shown that each set $I_{k}^{\prime}$ is a finite union of disjoint intervals $\left\{I_{i, k}^{\prime \prime}: i=1, \ldots, N_{k}\right\}$. Set $I_{i, k}=$ Interior $I_{i, k}^{\prime \prime}$; since $m$ is non-atomic the class of sets $\mathcal{I}=\left\{I_{i, k}: i=\right.$ $\left.1, \ldots, N_{k}, k \in \mathbb{N}\right\}$ has the desired property.

\section{MAIn RESUlts}

Theorem 1. Assume $m_{\mathcal{P}}$ satisfies the density theorem (in particular, if it is absolutely continuous with respect to the Lebesgue measure). Then the following conditions are equivalent:

a) $m_{\mathcal{P}}\left\{t: \Gamma^{\mathcal{T}_{D}}\right.$ is not a singleton $\}=0$.

b) $A=\int \psi(t) d P_{t}$ for $\psi$ a Borel function.

If these conditions hold, then $\psi=\psi_{\Gamma^{\mathcal{T}_{D}}} m_{\mathcal{P}}$-a.e.

Proof. a) $\Rightarrow$ b). To avoid overburdened notation, we shall not make explicit the dependence on $\mathcal{T}_{D}$. First observe that $\psi_{\Gamma}=\phi_{\Gamma}$ on $S(\Gamma)$, so the restriction $\psi_{\Gamma}$ : $S(\Gamma) \rightarrow \mathbb{R}$ is $\mathcal{T}_{D} / \mathcal{T}_{0}$-continuous. Using Lemma 2 , we get that $\forall \varepsilon>0$ and $\forall t \in S(\Gamma)$ there exists a $\mathcal{T}_{D}$ open set $I_{t} \in \mathcal{I}(t)$ such that

$$
\sigma\left(A P_{I_{t}}\right) \subset\left(\psi_{\Gamma}(t)-\varepsilon, \psi_{\Gamma}(t)+\varepsilon\right) ;
$$

hence, $\forall s \in I_{t} \cap S(\Gamma): \psi_{\Gamma}(s) \in\left(\psi_{\Gamma}(t)-\varepsilon, \psi_{\Gamma}(t)+\varepsilon\right)$.

Let $\mathcal{K}$ be the class of sets with respect to which $m_{\mathcal{P}}$ satisfies the density theorem. Take $\left\{K_{n}\right\} \in \mathcal{K}(t)$. There exists $n(\varepsilon)$ such that

$$
\forall n \geq n(\varepsilon): \quad \frac{m_{\mathcal{P}}\left(K_{n} \cap I_{t}\right)}{m_{\mathcal{P}}\left(K_{n}\right)} \geq 1-\varepsilon .
$$

We denote by $\psi$ any $\mathcal{L}_{\mathcal{P}}$-measurable extension of $\psi_{\Gamma}$ to $\mathbb{R}$ bounded by $\|A\|$. Let us take $f \in H$ such that $\left|\frac{d m_{f}}{d m_{\mathcal{P}}}\right| \leq c$. We have

$$
\begin{gathered}
\left|\left\langle A P_{K_{n}} f, f\right\rangle-\psi_{\Gamma}(t)\left\langle P_{K_{n}} f, f\right\rangle\right| \\
\leq\left|\left\langle A P_{K_{n} \cap I_{t}} f, f\right\rangle-\psi_{\Gamma}(t)\left\langle P_{K_{n} \cap I_{t}} f, f\right\rangle\right|+2\|A\|\left\|P_{K_{n} \backslash I_{t}} f\right\|^{2} \\
\leq \varepsilon \int_{K_{n} \cap I_{t}} \frac{d m_{f}}{d m_{\mathcal{P}}} d m_{\mathcal{P}}+2\|A\| \int_{K_{n} \backslash I_{t}} \frac{d m_{f}}{d m_{\mathcal{P}}} d m_{\mathcal{P}} \\
\leq \varepsilon c m_{\mathcal{P}}\left(K_{n}\right)+2\|A\| c \varepsilon m_{\mathcal{P}}\left(K_{n}\right)=c \varepsilon(2\|A\|+1) m_{\mathcal{P}}\left(K_{n}\right) .
\end{gathered}
$$


Hence:

$$
\left|\frac{1}{m_{\mathcal{P}}\left(K_{n}\right)}\left\langle A P_{K_{n}} f, f\right\rangle-\psi_{\Gamma}(t) \frac{1}{m_{\mathcal{P}}\left(K_{n}\right)}\left\langle P_{K_{n}} f, f\right\rangle\right| \leq c(2\|A\|+1) \varepsilon .
$$

From the density theorem, we get

$$
\left|\frac{d}{d m_{f}}\left\langle A P_{t} f, f\right\rangle-\psi_{\Gamma}(t) \frac{d}{d m_{f}}\left\langle P_{t} f, f\right\rangle\right| \leq c(2\|A\|+1) \varepsilon \quad m_{\mathcal{P}} \text {-a.e in } t .
$$

We conclude that

$$
\frac{d}{d m_{f}}\left\langle A P_{t} f, f\right\rangle=\psi_{\Gamma}(t) \frac{d}{d m_{f}}\left\langle P_{t} f, f\right\rangle \quad m_{\mathcal{P}} \text {-a.e. on } S(\Gamma) .
$$

Since $m_{\mathcal{P}}\left(S(\Gamma)^{c}\right)=0$, for any $f$ with $\left(\frac{d m_{f}}{d m_{\mathcal{P}}}\right)$ bounded we deduce that

$$
\langle A f, f\rangle=\int \psi_{\Gamma}(t) d\left\langle P_{t} f, f\right\rangle
$$

By standard density arguments we obtain this last equality for any $f \in H$.

b) $\Rightarrow$ a). Let $A=\int \psi(t) d P_{t}$ with $\psi$ Borel measurable. $\psi$ is $\mathcal{T} / \mathcal{T}_{0}$-continuous $m_{\mathcal{P}}$-a.e.; that is, there exists a full $m_{\mathcal{P}}$-measurable set $E \subseteq F_{\mathcal{P}}$ such that for any $t \in E, \varepsilon>0$ there exists $I_{t} \in \mathcal{I}(t)$ such that $\forall s \in I_{t},|\psi(s)-\psi(t)| \leq \varepsilon$. It is easily obtained that:

$$
\sigma\left(A P_{I_{t}}\right) \subset[\psi(t)-\varepsilon, \psi(t)+\varepsilon] .
$$

As this happens for any $\varepsilon>0$, we deduce that for any $t \in E, \Gamma(t)=\{\psi(t)\}$. Then $S\left(\Gamma^{\mathcal{T}_{D}}\right) \supset E$, and it is a full $m_{\mathcal{P}}$-measurable set.

Theorem 2. The following two conditions are equivalent:

a) $m_{\mathcal{P}}\left\{t: \Gamma^{\mathcal{T}_{0}}\right.$ is not a singleton $\}=0$.

b) For any $\varepsilon>0$ there exists a class of disjoint open intervals $\mathcal{I}_{\varepsilon}=\left\{I_{n, \varepsilon}: n \in \mathbb{N}\right\}$ of full $m_{\mathcal{P}}$-measure, i.e. $m_{\mathcal{P}}\left(\left(\bigcup_{n \in \mathbb{N}} I_{n, \varepsilon}\right)^{c}\right)=0$, and a real sequence $\left(c_{n, \varepsilon}: n \in \mathbb{N}\right)$ such that

$$
\forall f \in H, \quad\left|\langle A f, f\rangle-\sum_{n \in \mathbb{N}} c_{n, \varepsilon}\left\langle P_{I_{n, \varepsilon}} f, f\right\rangle\right| \leq \varepsilon\|f\|^{2} .
$$

Moreover, when these conditions hold we have that for any $\varepsilon>0$ we can choose a covering $\mathcal{I}_{\varepsilon}$ of $S_{\Gamma}^{\mathcal{T}_{0}}$ such that each element of $\mathcal{I}_{\varepsilon}$ intersects $S_{\Gamma}^{\mathcal{T}_{0}}$, and $c_{n, \varepsilon}=\Gamma^{\mathcal{T}_{0}}\left(\alpha_{n, \varepsilon}\right)$ with $\alpha_{n, \varepsilon} \in I_{n, \varepsilon} \cap S_{\Gamma}^{\mathcal{T}_{0}}$. In particular, $m_{\mathcal{P}}\left(\left(S_{\Gamma}^{\mathcal{T}_{0}}\right)^{c}\right)=0$ implies $A=\int_{S_{\Gamma}} \mathcal{T}_{0} \Gamma(t) d P_{t}$.

Proof. As before, we shall not make explicit the dependence on $\mathcal{T}_{0}$. Assume b) holds. Take $D=\bigcap_{k \geq 1} \bigcup_{n \in \mathbb{N}} I_{n, \frac{1}{k}}$. Then $m_{\mathcal{P}}\left(D^{c}\right)=0$. Fix $t \in D$. For any $k \geq 1$ there exists some $n_{k} \in \mathbb{N}$ such that $t \in I_{n_{k}, \frac{1}{k}}$. Denote $I=I_{n_{k}, \frac{1}{k}}, c=c_{n_{k}, \frac{1}{k}}$. Then

$$
\left|\left\langle A P_{I} f, f\right\rangle-c\left\langle P_{I} f, f\right\rangle\right| \leq \frac{1}{k}\left\|P_{I} f\right\|^{2} \text {. }
$$

Hence $\sigma\left(A P_{J}\right) \subseteq\left(c-\frac{1}{k}, c+\frac{1}{k}\right)$ for any $J \subset I, J \in \mathcal{I}_{t}$. Then the diameter of $\Gamma(t)$ satisfies $\delta(\Gamma(t)) \leq \frac{2}{k}$. We deduce that $\Gamma(t)$ is a singleton, so $D \subset S(\Gamma)$ and $m_{\mathcal{P}}\left(S(\Gamma)^{c}\right) \leq m_{\mathcal{P}}\left(D^{c}\right)=0$.

Now assume condition a) holds, and fix $\varepsilon>0$. For any $t \in S(\Gamma)$ there exists $\delta(t)>0$ such that for $J_{t}=(t-\delta(t), t+\delta(t))$ we have $\sigma\left(A P_{J_{t}}\right) \subset\left(\Gamma(t)-\frac{\varepsilon}{2}, \Gamma(t)+\frac{\varepsilon}{2}\right)$. At this point we notice that $m_{\mathcal{P}}\left(J_{t}\right)>0$. Consider $\mathcal{J}=\left\{J_{t}: t \in S(\Gamma)\right\}$. By Lemma 5 there exists a countable class of disjoint open intervals $\mathcal{I}=\left\{I_{n}: n \in \mathbb{N}\right\}$ 
subordinated to $\mathcal{J}$ and which $m_{\mathcal{P}}$-covers $S(\Gamma)$. Since $m_{\mathcal{P}}\left(S(\Gamma)^{c}\right)=0$, we may assume that $m_{\mathcal{P}}\left(I_{n} \cap S(\Gamma)\right)>0$ for any $n \in \mathbb{N}$. Take $\alpha_{n} \in I_{n} \cap S(\Gamma)$; we have

$$
\left|\langle A f, f\rangle-\sum_{n \in \mathbb{N}} \Gamma\left(\alpha_{n}\right)\left\langle P_{I_{n}} f, f\right\rangle\right| \leq \sum_{n \in \mathbb{N}}\left|\left\langle A P_{I_{n}} f, f\right\rangle-\Gamma\left(\alpha_{n}\right)\left\langle P_{I_{n}} f, f\right\rangle\right| .
$$

Since $\sigma\left(A P_{I_{n}}\right)$ is the spectrum of $A P_{I_{n}}$ on $P_{I_{n}} H$, we have

$$
\begin{aligned}
& \left|\left\langle A P_{I_{n}} f, f\right\rangle-\Gamma\left(\alpha_{n}\right)\left\langle P_{I_{n}} f, f\right\rangle\right| \\
& \quad \leq \sup \left\{\left|\lambda-\Gamma\left(\alpha_{n}\right)\right|: \lambda \in \sigma\left(A P_{I_{n}}\right)\right\}\left\langle P_{I_{n}} f, f\right\rangle .
\end{aligned}
$$

For any $n \in \mathbb{N}$ we have $I_{n} \subset J_{t}$ for some $t \in S(\Gamma)$. Since $\alpha_{n} \in I_{n} \subset J_{t}$, we have $\left|\Gamma\left(\alpha_{n}\right)-\Gamma(t)\right|<\frac{\varepsilon}{2}$. On the other hand, for any interval $J$ with $m_{\mathcal{P}}(J)>0$ and $J \subset J_{t}$ we have $\sigma_{J} \subset \sigma_{J_{t}} \subset\left(\Gamma(t)-\frac{\varepsilon}{2}, \Gamma(t)+\frac{\varepsilon}{2}\right)$. Then the right hand side of (2) is bounded by $\varepsilon\left\langle P_{I_{n}} f, f\right\rangle$ and the right hand side of (1) is bounded by $\varepsilon \sum_{n \in \mathbb{N}}\left\langle P_{I_{n}} f, f\right\rangle=\varepsilon\langle f, f\rangle$, because $m_{f}\left(S(\Gamma)^{c}\right)=0$. We have shown that

$$
\left|\langle A f, f\rangle-\sum_{n \in \mathbb{N}} \Gamma\left(\alpha_{n}\right)\left\langle P_{I_{n}} f, f\right\rangle\right| \leq \varepsilon\langle f, f\rangle .
$$

Let us show the last statement. We take $\alpha_{n, \varepsilon}, I_{n, \varepsilon}$ instead of $\alpha_{n}, I_{n}$ because these quantities depend on $\varepsilon>0$. Since $\Gamma$ restricted to $S_{\Gamma}$ is $\mathcal{T}_{0} / \mathcal{T}_{0}$-continuous, we get

$$
\sum_{n \in \mathbb{N}} \Gamma\left(\alpha_{n, \varepsilon}\right) \mathbb{1}_{I_{n, \varepsilon}}(t) \underset{\varepsilon \rightarrow 0}{\longrightarrow} \Gamma(t) \quad m_{\mathcal{P}} \text {-a.e. on } S_{\Gamma} \text {. }
$$

On the other hand, $\left|\sum_{n \in \mathbb{N}} \Gamma\left(\alpha_{n, \varepsilon}\right) \mathbb{1}_{I_{n, \varepsilon}}\right| \leq\|A\|$, so by the dominated convergence theorem we get

$$
\sum_{n \in \mathbb{N}} \Gamma\left(\alpha_{n, \varepsilon}\right)\left\langle P_{I_{n, \varepsilon}} f, f\right\rangle \underset{\varepsilon \rightarrow 0}{\longrightarrow} \int_{S_{\Gamma}} \Gamma(t) d\left\langle P_{t} f, f\right\rangle .
$$

Example. Let $A$ be a self-adjoint operator commuting with $\mathcal{P}$. It is said that $\mathcal{P}$ separates the spectrum (see [4]) of $A$ if there exists an increasing function $g(t)$ such that

$$
\sigma_{(-\infty, t]} \subset(-\infty, g(t)], \quad \sigma_{[t, \infty)} \subset[g(t), \infty) .
$$

In this case we have $\sigma_{[s, t]} \subseteq[g(s), g(t)]$. We deduce that $\Gamma^{\mathcal{T}_{0}}(t) \subseteq[g(t-), g(t+)]$. As $g$ is increasing and the maximal spectral type measure $m_{\mathcal{P}}$ is non-atomic, we get

$$
m_{\mathcal{P}}\left\{t: \Gamma^{\mathcal{T}_{0}}(t) \text { is not a singleton }\right\}=0
$$

Moreover, $\Gamma^{\mathcal{T}_{0}}(t)=\{g(t)\} m_{\mathcal{P}}$-a.e.

\section{COMPLEMENT TO THIS PAPER}

In what follows we describe, using the pointwise spectrum, the least upper bound of $A$ in $\mathcal{A}_{\mathcal{P}}$ : the closed algebra of symmetric operators generated by $\mathcal{P}$. Notice that there is a unique symmetric operator $A^{+} \in \mathcal{A}_{\mathcal{P}}$, which satisfies

1) $A^{+} \geq A$

2) $\forall B \in \mathcal{A}_{\mathcal{P}}$, if $B \geq A$, then $B \geq A^{+}$. 
In fact since $\mathcal{A}_{\mathcal{P}}$ is a closed lattice it is easy to prove that

$$
A^{+}=\int \alpha_{A}(t) d P_{t}
$$

where

$$
\alpha_{A}=\operatorname{ess} \inf \left\{h / h \in \mathcal{L}_{P} \text { and } \int h(t) d P_{t} \geq A\right\} .
$$

Here the essinf is computed with respect to $m_{\mathcal{P}}$.

The following result characterizes $\alpha_{A}$ in terms of $\Gamma_{A}^{\mathcal{T}_{D}}$.

Theorem. If $m_{\mathcal{P}}$ satisfies a density theorem, then

$$
\alpha_{A}=\max \Gamma_{A}^{\mathcal{T}_{D}} \quad m_{\mathcal{P}}-a . e .
$$

Proof. We denote $\psi=\max \Gamma_{A}^{\mathcal{T}_{D}}(t)$ which is $\mathcal{L}_{\mathcal{P}}$-measurable by Lemma 3 . From Theorem 1 we have $\Gamma_{A^{+}}^{\mathcal{T}_{D}}(t)=\left\{\alpha_{A}(t)\right\} m_{\mathcal{P}}$-a.e. In that way for any point $t \in F_{\mathcal{P}}$ of continuity for $\alpha_{A}$ we get

$$
\forall \varepsilon>0 \exists I \in \mathcal{I}^{\mathcal{T}_{D}}(t) \forall s \in I \alpha_{A}(s) \leq \alpha_{A}(t)+\varepsilon .
$$

Since

$$
\begin{aligned}
\left\langle A P_{I} f, f\right\rangle & \leq\left\langle A^{+} P_{I} f, f\right\rangle=\int_{I} \alpha_{A}(s) d\left\langle P_{s} f, f\right\rangle \\
& \leq\left(\alpha_{A}(t)+\varepsilon\right)\left\langle P_{I} f, f\right\rangle
\end{aligned}
$$

we conclude $\Gamma_{A}^{\mathcal{T}_{D}}(t) \subseteq\left(-\infty, \alpha_{A}(t)+\varepsilon\right]$, and therefore $\psi(t) \leq \alpha_{A}(t)$. Almost all points in $F_{\mathcal{P}}$ are continuity points for $\alpha_{A}$ (due to the density theorem) from which we conclude

$$
\psi \leq \alpha_{A} \quad m_{\mathcal{P}} \text {-a.e. }
$$

In order to prove the opposite inequality it is enough to show that $\int \psi(t) d P_{t} \geq A$. For that purpose consider $f \neq 0$, a fixed element in $H$, and introduce the following signed measure on $\mathcal{L}_{\mathcal{P}}, \nu_{f}(\Lambda)=\left\langle A P_{\Lambda} f, f\right\rangle$. We have $\nu_{f}<<m_{f}$ and moreover $\left|\frac{d \nu_{f}}{d m_{f}}\right| \leq\|A\|$. Consider also $\eta=\frac{d m_{f}}{d m_{\mathcal{P}}}$.

We have that $\eta$ is $\mathcal{T}_{D} / \mathcal{T}_{0}$-continuous $m_{\mathcal{P}}$-a.e. Let $G=\left\{t \in F_{\mathcal{P}} / \eta(t)>0\right.$ and $\eta$ is continuous on $t\}$; then $m_{f}\left(G^{c}\right)=0$. Now if $I \in \mathcal{T}_{D}$ and $I \cap G \neq \varnothing$, then $m_{f}(I)>0$. In fact let $t \in I \cap G$; since $\eta$ is continuous on $t$, there exists an open neighbourhood $J \subseteq I$ of $t$ such that $\eta(s)>0$ for all $s \in J$. Therefore, since $m_{\mathcal{P}}(J)>0$,

$$
\nu_{f}(I) \geq \nu_{f}(J)=\int_{J} \eta(s) d m_{\mathcal{P}}(s)>0 .
$$

Almost all points $t \in G$ (with respect to $m_{f}$ or $m_{\mathcal{P}}$ ) are continuity points for $\frac{d \nu_{f}}{d m_{f}}$. Fix one such $t \in G$; from Lemma 2 we conclude that

$$
\begin{aligned}
\forall \varepsilon>0 & \exists I \in \mathcal{I}^{\mathcal{T}_{D}}(t) \text { such that } \\
& \sigma\left(A P_{I}\right) \subseteq(-\infty, \psi(t)+\varepsilon) \text { and } \\
& \forall s \in I\left|\frac{d \nu_{f}(s)}{d m_{f}}-\frac{d \nu_{f}(t)}{d m_{f}}\right| \leq \varepsilon .
\end{aligned}
$$

Hence, $\forall g \in H\left\langle A P_{I}, g\right\rangle \leq(\psi(t)+\varepsilon)\left\langle P_{I} g, g\right\rangle$. In particular, if $g=P_{\Lambda} f$, where $\Lambda \subseteq I$, we have

$$
\nu_{f}(\Lambda)=\left\langle A P_{\Lambda} f, f\right\rangle \leq(\psi(t)+\varepsilon)\left\langle P_{\Lambda} f, f\right\rangle=(\psi(t)+\varepsilon) m_{f}(\Lambda)
$$


and therefore $\frac{d \nu_{f}(s)}{d m_{f}} \leq(\psi(t)+\varepsilon) m_{f}$-a.e. on $s \in I$. Since $m_{f}(I)>0$ we get that $\frac{d \nu_{f}(t)}{d m_{f}} \leq \psi(t)+2 \varepsilon$. We have proved that $\frac{d \nu_{f}}{d m_{f}} \leq \psi m_{f}$-a.e.

Finally,

$$
\langle A f, f\rangle=\int \frac{d \nu_{f}(t)}{d m_{f}} d m_{f}(t) \leq \int \psi(t) d\left\langle P_{t} f, f\right\rangle
$$

as we wanted to prove.

In a similar way the operator $A^{-}=\int \phi(t) d P_{t}$ with $\phi(t)=\min \Gamma_{A}^{\mathcal{T}_{D}}(t)$ is the greatest lower bound of $A$ in $\mathcal{A}_{\mathcal{P}}$.

\section{ACKNOWLEDGMEnTs}

This work was partially supported by FONDECYT.

\section{REFERENCES}

1. N.I. Akhiezer and I.M. Glazman. (1961). Theory of Linear Operators in Hilbert Space, Vol. I, II, Frederick Ungar Publ. Co., New York. MR 41:9015

2. J.P. Aubin and A. Cellina. (1984). Differential Inclusions, Springer-Verlag, Berlin. MR 85j: 49010

3. C. Goffman, C.J. Nengebauer and T. Nishiura (1961). Density topology and approximate continuity. Duke Math. J., 28, 497-506. MR 25:1254

4. I.T. Gohberg and M.G. Krein. (1967). Description of contraction operators which are similar to unitary operators. Func. Anal. Appl. 1, 33-52. MR 35:4761

5. P. Masani and M. Rosenberg. (1976). When is an operator the integral of a given spectral measure? J. of Functional Analysis 21, 88-121. MR 53:6347

6. N. Martin. A topology for certain measure spaces (1964). Transactions Amer. Math. Soc., 112, 1-18; 114 (1965), 280. MR 28:5151; MR 30:2113

7. S. Scheinberg. (1971). Topologies which generate a complete measure algebra. Adv. in Math. 7, 231-239. MR 44:4172

8. E. Stein. (1970). Singular Integrals and Differentiability Properties of Functions. Princeton Univ. Press. MR 44:7280

(N. Bertoglio) Facultad de Matemática, Pontificia Universidad Católica de Chile, Casilla 306, Correo 22, Santiago, Chile

E-mail address: nbertogl@riemann.mat.puc.cl

(S. Martínez and J. San Martín) Universidad de Chile, Facultad de Ciencias Físicas y Matemáticas, Departamento de Ingeniería Matemática, Casilla 170-3, Correo 3, SantiAGo, ChILE

E-mail address: smartine@dim.uchile.cl

E-mail address: jsanmart@dim.uchile.cl 